\title{
Communicative Participation in People with Amyotrophic Lateral Sclerosis
}

\author{
Malin Sixt Börjesson ${ }^{a, b}$ Lena Hartelius ${ }^{b}$ Katja Laakso $^{b}$ \\ ${ }^{a}$ Department of Neurologopedics, Sahlgrenska University Hospital, Gothenburg, Sweden; ${ }^{b}$ Speech and Language \\ Pathology Unit, Institute of Neuroscience and Physiology, University of Gothenburg, Gothenburg, Sweden
}

\section{Keywords}

Communicative participation - Communicative

Participation Item Bank · Amyotrophic lateral sclerosis .

Dysarthria $\cdot$ Intelligibility

\begin{abstract}
Background: Communication is affected in most people with amyotrophic lateral sclerosis (ALS); up to $80-95 \%$ will reach a point where they are no longer able to meet their communicative needs with natural speech. The deterioration of speech and communicative abilities presumably has an impact on communicative participation. However, little is known about how these factors relate to each other in this population of patients. Objective: This study aimed to investigate the association between communicative participation, functional deficits, and severity of dysarthria in individuals with ALS. Method: Thirty people with ALS were rated for (1) communicative participation, using the Communicative Participation Item Bank (CPIB, Swedish); and (2) disability related to the disease, using the Revised ALS Functional Rating Scale (Swedish). An expert listening panel assessed intelligibility and severity of dysarthria based on recorded text readings and sentences from the Swedish Test of Intelligibility. Results: CPIB scores were significantly lower for participants with moderate/severe dysarthria than for those with no/
\end{abstract}

\begin{tabular}{ll}
\hline karger@karger.com & $\begin{array}{l}\text { (C) } 2020 \text { The Author(s) } \\
\text { Published by S. Karger AG, Basel }\end{array}$ \\
www.karger.com/fpl & This article is licensed under the Creative Commons Attribution- \\
Karger & $\begin{array}{l}\text { NonCommercial-NoDerivatives 4.0 International License (CC BY- } \\
\text { NC-ND) (http://www.karger.com/Services/OpenAccessLicense). } \\
\text { Usage and distribution for commercial purposes as well as any dis- } \\
\text { tribution of modified material requires written permission. }\end{array}$
\end{tabular}

mild dysarthria and correlated with bulbar function and intelligibility. Conclusion: The study found that the CPIB provides a means to rate and discuss communicative participation with persons with ALS, which could assist in the planning of further efforts/services.

(C) 2020 The Author(s)

Published by S. Karger AG, Basel

\section{Introduction}

Amyotrophic lateral sclerosis (ALS) is a motor neuron disease characterized by progressive muscular paralysis, reflecting the degeneration of upper and lower motor neurons $[1,2]$. Dysarthria is the first or most prominent sign in early-stage ALS in $25-30 \%$ of patients. The involvement of both upper and lower motor neurons usually results in a mixed dysarthria with both spastic and flaccid components [3]. Communication is affected at some time during the course of the disease in most people with ALS. Up to $80-95 \%$ of all people with ALS eventually reach a point where they are no longer able to meet their communicative needs with natural speech [4].

This study was part of a project at the Speech and Language Pathology Unit at the University of Gothenburg in collaboration with the Karolinska Institute, Stockholm. 
In the International Classification of Functioning, Disability and Health (ICF), participation refers to the nature and extent of an individual's involvement in life situations. Participation restrictions are the difficulties that individuals can experience in life situations due to a health condition $[5,6]$. Dykstra [7] wrote that "within the realm of communication, participation refers to the roles and activities that one chooses which involve communication within the context of daily life" (p. 403). Communicative participation has been defined by Eadie et al. [8] as "taking part in life situations where knowledge, information, ideas, and feelings are exchanged."

Dysarthria is characterized by slow, weak, imprecise, and/or uncoordinated movements of the speech musculature [9]. A variety of measures are used to assess dysarthria. Intelligibility testing through transcription of a set of words and/or phrases by unfamiliar listeners has been used as a somewhat objective measure [10]. Whereas intelligibility refers to how accurately a speaker's acoustic signal is received by the listener, comprehension refers to the listener's ability to interpret the meaning of a spoken message. Sussman and Tjaden [11] found that scaled estimates of dysarthria severity appear to be sensitive to aspects of speech impairment that are not captured by sentence intelligibility scores, e.g., voice quality, articulation, prosody, and overall comprehension. Dysarthria can have a negative impact on communicative participation, e.g., through the loss of intelligibility and thereby lessened communicative effectiveness, or through communicative apprehension, i.e., a heightened level of communicationrelated anxiety [12]. Dysarthria is often associated with chronic neurodegenerative diseases. These conditions are associated with additional symptoms that may interfere with communicative participation. For example, facial or arm/hand muscle weakness, or paralysis, can impair communication directly through decreased facial expressions and gesturing. Communicative participation can also be affected indirectly, e.g., if lower-limb musculature, sight, and/or balance are affected, making it harder to access communicative situations and settings. To find out more about the similarities and differences in communicative participation in different communication disorders, a qualitative study was conducted where self-report data were collected from 44 individuals representing 7 different medical diagnoses: spasmodic dysphonia, multiple sclerosis, stroke, Parkinson's disease (PD), stuttering, ALS, and laryngectomy. The study found that despite differences in impairment and activity limitations, participants described similar communicative participation restrictions [13].
The relationship between dysarthria and communicative participation is not necessarily straightforward. For example, in a study by Hartelius et al. [14], people with moderate dysarthria reported more communicative difficulties than other severity groups. McAuliffe [15] speculates that "an individual's perception of their speech production may have greater influence on communicative participation than the result of objective measurements of the adequacy of speech" (p. 412). Communicative participation has been found to be associated with fatigue in $\mathrm{PD}$, cognitive/communication skills in multiple sclerosis, and swallowing in ALS, as well as with speech severity and speech usage in all 3 diseases [15-17]. Communicative participation may not only be related to a person's skill set, but also, for example, to the attitude of communication partners and whether the person uses a communication aid, e.g., a speech-generating device. Thus, participation can be affected by factors within a person, such as structural or functional deficits or personality, and environmental factors.

In a review of self-report instruments in speech-language pathology, Eadie et al. [8] found that several of the reviewed instruments measured aspects of the construct of communicative participation, but that none of them measured the construct exclusively. A number of suggestions were given for how to design a future instrument to ensure that it would measure the full scope of this specific construct, e.g., that it would have sound psychometric properties, that it would be acceptable to respondents, and that it would be clinically useful for measuring therapy outcomes, all suggestions that the same research group adhered to in developing the Communicative Participation Item Bank (CPIB).

The CPIB is a self-report outcome measurement tool. It is intended for measuring communicative participation in a community-dwelling adult population, and it asks questions about the extent to which a person feels restricted when communicating in various situations. It has been developed using item response theory (IRT). IRT estimates the relationship among the characteristics of items in an instrument, how the individual responds to each item, and that particular individual's underlying latent trait (i.e., a variable that cannot be measured directly, in this case, communicative participation) [18]. A large set of candidate items was reduced to 46 items that demonstrate strong evidence of unidimensionality, local independence, good item fit, and good measurement precision $[19,20]$. These items concern how much a person's condition interferes in typical speaking situations, such as asking questions in a conversation, giving someone de- 
tailed information, or communicating in a small group of people. A 10-item general short form was generated from the 46 -item bank. It was designed to capture a wide range of communication situations while keeping the response burden low. The development of the CPIB and general short form has been described in great detail elsewhere $[19,20]$. The CPIB has been thoroughly tested on participants with mild-to-moderate dysarthria.

In calibrating the items and finalizing the CPIB, selfreport data were collected from 701 individuals and involving 4 diagnoses (multiple sclerosis, PD, ALS, and head and neck cancer). While people with ALS were included in the initial instrument calibration sample, due to their small numbers these individuals were not included specifically in the differential item functioning (DIF) analysis to demonstrate commensurate measurement properties across disorders. The DIF analysis showed no meaningful differences across the other 3 diagnostic groups [18]. In subsequent studies using the CPIB with other diagnoses, it has been found that "symptoms of various disorders interact with common barriers (...) leading to shared restrictions in communicative participation (...)" [21] (p. 693). The 46-item CPIB was translated to Swedish in 2012. The translation has been tested and adapted through cognitive interviews in 2 separate graduate papers, one using respondents with dysarthria following multiple sclerosis and/or ataxia, and the other using respondents with dysarthria following PD or atypical Parkinsonism [22, 23]. Baylor et al. [24] compared data from 200 people in New Zealand (NZ) and 200 in the USA with PD, using DIF to investigate whether there is a cultural bias in the CPIB. No items were identified as having a statistically significant DIF across the US and NZ cohorts, thus indicating that the CPIB items and scoring parameters are also suitable for respondents from NZ.

This study was designed to investigate how the CPIB relates to different functional deficits caused by ALS and different severity levels of dysarthria. The aim was to explore the following hypotheses:

1. Individuals with increased functional deficits caused by ALS (specifically lessened bulbar function) will perceive decreased communicative participation;

2. Individuals with ALS and lower speech intelligibility will perceive decreased communicative participation compared with individuals with higher speech intelligibility;

3. Individuals with ALS and moderate/severe dysarthria will perceive decreased communicative participation compared with individuals with ALS and no/mild dysarthria.

Communicative Participation in People with ALS

\section{Materials and Methods}

This exploratory correlational study, involving a convenience sample of 30 people with ALS and a listening panel consisting of 5 speech-and-language pathologists (SLPs), was designed to investigate how the deterioration of speech and communicative abilities relates to communicative participation.

\section{Participants}

The inclusion criteria were: an age of $\geq 18$ years, Swedish language fluency, living at home, and having a diagnosis of ALS made by a neurologist. The exclusion criteria were: dementia or other cognitive deficits to a degree that would interfere with participation in the study, and impaired sight or hearing. People in a palliative stage of disease were also excluded. Dysarthria was not in itself an inclusion criterion, nor was severe dysarthria used as an exclusion criterion. Outpatients who were judged by their ALS team in consensus to fit the criteria were invited to participate in the study.

The study included a total of 30 participants ( 14 women and 16 men) who were 43-77 years old. Twenty-three participants were from the caseload of the ALS team at Sahlgrenska University Hospital and 7 were connected to other hospitals in the western region of Sweden. All participants were community-dwelling except for 1 person on home ventilation who, because of age-related regulations, could not be granted personal assistance and therefore resided in a home for the elderly. In line with previous studies and since the CPIB has not yet been tested with people using alternative and augmentative communication (AAC), the participants had to use oral speech as their primary mode of communication at the time of inclusion. However, at the time of testing, 2 participants communicated mostly using AAC. One used both high-tech AAC in the form of a computer tablet and a cell phone and low-tech $\mathrm{AAC}$ in the form of writing, gestures, and using her husband as spokesperson and interpreter; the other wrote through a proxy on a laptop. Table 1 presents participant demographics.

\section{Material and Procedure}

The CPIB consists of 46 items asking respondents to rate how much their condition interferes with communicative participation in a variety of life situations on a 4 -point Likert scale ( $3=$ not at all, $2=$ a little, 1 = quite a bit, $0=$ very much). The general 10 -item short form includes items concerning to what extent the condition interferes with "talking to people you do not know," "communicating in a small group of people," and "getting your turn in a fastmoving conversation." For the short form, the summary score ranges from 0 to 30 , with high scores indicating less interference with communicative participation. A score translation table has been generated using the 10 -item short form items, including a conversion to standard $\mathrm{T}$ scores $($ mean $=50$; standard deviation $=$ 10, range 24.20-71) [20]. For this study, the adapted version in Swedish was used $[22,23]$. The CPIB 10-item short form answers were pulled from the responses to the full 46 -item bank.

The Revised Amyotrophic Lateral Sclerosis Functional Rating Scale (ALSFRS-R) [25] has been and is currently used as a primary or secondary outcome measure in multiple ALS clinical trials. It consists of 12 items that assess physical function in carrying out the activities of daily living in 4 domains: bulbar functions (speech, salivation, and swallowing), fine-motor tasks (handwriting, cutting food/handling percutaneous nutrition, dressing, and hygiene), gross-motor tasks (turning in bed, walking, and climbing 
Table 1. Participant demographics

\begin{tabular}{lc}
\hline Age, years & $61.3 \pm 8.2(43-77)$ \\
Male & $16(53.3)$ \\
Female & $14(46.7)$ \\
Disease duration, years & $4.6(0.5-28 ; 2.7)$ \\
Duration of speech difficulties, years & 2.8 years $(0-28 ; 1)$ \\
Degree of professional activity & \\
$\quad$ Not working & $21(70)$ \\
$\quad$ Working part-time & $6(20)$ \\
$\quad$ Working full-time & $3(10)$ \\
\hline
\end{tabular}

Values express $n(\%)$, mean \pm SD (range), or mean (range; median)

stairs), and respiratory function (dyspnea, orthopnea, and respiratory insufficiency). Four of the response options of item 1 (speech) describe dysarthria of increasing severity, and the response options of both item 2 (salivation) and item 3 (swallowing) include aspects of oral motor function. Thus, a relation between the bulbar domain of the ALSFRS-R and both intelligibility and dysarthria severity can be surmised. The scale has shown strong internal consistency and construct validity, and has been found to correlate with objective measures of muscle strength and pulmonary function and quality-of-life measures [26], although a more recent investigation of the scale showed that it might benefit from a modification of its response options to increase sensitivity across the spectrum of ALS progression [27]. The ALSFRS-R was used in a Swedish translation similar to a version used by Ozanne and Persson [28]. Functions according to the ALSFRS-R were assessed together with the participant.

The speech assessment in this study included measures of both intelligibility and the severity of dysarthria. Intelligibility was measured on the sentence level (\% correctly identified content words), where each participant read a unique list of 10 computer-generated nonsense sentences from the Swedish Test of Intelligibility (STI) [29], with the instruction to read "as if this were something you would say." The recorded STI sentences and text readings were deidentified, and approximately $23 \%$ of the samples were reduplicated for the assessment of intrarater reliability. Five highly experienced SLPs, with extensive experience assessing adult patients with dysarthria, were asked to orthographically transcribe the lists of STI sentences after a maximum of 2 presentations of each sentence. The STI scores were averaged across all 5 SLP assessments for the final score used in the study. The SLP listening panel was also asked to grade the severity of dysarthria on a 4-point scale, as perceived from a recorded text sample, of which they were permitted unlimited presentations. The scale points were $(0)$ none, (1) mild, (2) moderate, and (3) severe. The median severity grade was used for the statistical calculations.

The first author was also the test leader. The entire test battery was administered in 20-75 min in a set order. It started with recordings and then moved on to the ALSFRS-R and demographic data. The CPIB was administered last. The speech assessments were recorded on a Zoom H2. The recording device was placed on a table directly in front of and approximately $50 \mathrm{~cm}$ from the participant. Some of the recordings were done at the home of the participant, and the placing of the device there depended on the environment and therefore varied. All participants except for one (no recording of the STI sentences was made for this individual) completed the entire test battery. Missing relevant data are reported in the results and tables.

\section{Statistics}

Descriptive statistics were used to describe and summarize the data. Because of the small group size and types of data collected, nonparametric tests were used. Intrarater reliability for the assessment of intelligibility was calculated using Spearman's rank-order correlation, and ranged from 0.77 to $0.94(p=0.009-0.000)$ for the 5 judges, with the correlation coefficient being used to measure the strength and direction of associations between the CPIB, ALSFRS$\mathrm{R}$, and STI. To avoid confounders due to small group sizes, participants were grouped into 2 severity levels, namely no/mild dysarthria and moderate/severe dysarthria, prior to statistical calculations according to the listening panel's assessment. A MannWhitney $U$ test was used to compare differences between the 2 dysarthria severity groups.

\section{Results}

The CPIB short form was completed by all 30 participants. T-scores were in the range of 34-71 points; high scores indicated less interference with communicative participation. Descriptive statistics show that the mean on the CPIB was 56.20 points (SD 10.94) and the median 57.15 points (range $34-71$ ).

The speech assessment by the listening panel showed that $17(56.7 \%)$ of the participants had no/mild dysarthria and 13 (43.3\%) had moderate/severe dysarthria. The participants with no/mild dysarthria had a mean intelligibility of $97.6 \%$ and a range of $93.78-100 \%$, while the participants with moderate/severe dysarthria had a mean intelligibility of $54.4 \%$ but a range of $3.1-99.1 \%$. Severity ratings made by all the listeners were the same for 15 of the participants (i.e., $6,3,2$, and 4 , respectively in the categories none, mild, moderate, and severe), and within 1 scale step for another 14 participants: no/mild $(n=7)$, mild/moderate $(n=5)$, and moderate/severe $(n=7)$ dysarthria. Hence, 29 of the 30 ratings were within 1 scale step.

For people with no/mild dysarthria, the mean CPIB score was 63.64 points (SD 5.63) and the median was 64.20 points (range $55.30-71$ ). For those with moderate/ severe dysarthria, the mean CPIB score was 46.47 points (SD 8.11 ) and the median was 47.80 points (range $34-$ 64.20). The lowest score was 34 points and 3 participants had a full score (71 points).

\section{Communicative Participation, the ALSFRS-R, and Speech Intelligibility}

Table 2 shows correlations between scores on communicative participation, ALSFRS-R, and speech intelligibil- 
Table 2. Correlation coefficients between communicative participation, the ALSFRS-R (Swedish) and its subsets, and speech intelligibility

\begin{tabular}{|c|c|c|c|c|c|c|}
\hline & \multirow{2}{*}{$\frac{\text { CPIB sf }}{\text { T-score }}$} & \multicolumn{5}{|c|}{ ALSFRS-R } \\
\hline & & $\begin{array}{l}\text { bulbar } \\
\text { score }\end{array}$ & $\begin{array}{l}\text { fine-motor } \\
\text { score }\end{array}$ & $\begin{array}{l}\text { gross-motor } \\
\text { score }\end{array}$ & $\begin{array}{l}\text { respiration } \\
\text { score }\end{array}$ & $\begin{array}{l}\text { total } \\
\text { score }\end{array}$ \\
\hline \multicolumn{7}{|l|}{ ALSFRS-R } \\
\hline Bulbar score & $0.764^{* *}$ & 1.000 & & & & \\
\hline Fine-motor score & -0.002 & -0.014 & 1.000 & & & \\
\hline Gross-motor score & -0.006 & -0.039 & $0.809^{* *}$ & 1.000 & & \\
\hline Respiration score & 0.237 & 0.273 & 0.209 & 0.287 & 1.000 & \\
\hline Total score & 0.244 & 0.323 & $0.757^{* *}$ & $0.804^{* *}$ & $0.667^{* *}$ & 1.000 \\
\hline STI $(n=29)^{1}$ & $0.517^{* *}$ & $0.789^{* *}$ & 0.261 & 0.144 & 0.265 & $0.418^{*}$ \\
\hline
\end{tabular}

CPIB sf, Communicative Participation Item Bank short form; ALSFRS-R, ALS Functional Rating ScaleRevised; STI, Swedish Test of Intelligibility. ${ }^{*}$ Correlation is significant at $p<0.05 .{ }^{* *}$ Correlation is significant at $p<0.01$.

${ }^{1}$ STI data were missing for one of the participants.

Table 3. Dysarthria severity and ALSFRS-R differences

\begin{tabular}{llll}
\hline ALSFRS-R & $\begin{array}{l}\text { No/mild } \\
\text { dysarthria } \\
(n=17)\end{array}$ & $\begin{array}{l}\text { Moderate/severe } \\
\text { dysarthria } \\
(n=13)\end{array}$ & $p$ value \\
\hline Bulbar items & 10.88 & 6.92 & $0.000^{*}$ \\
Fine-motor items & 6.88 & 5.46 & 0.363 \\
Gross-motor items & 6.53 & 5.23 & 0.536 \\
Respiratory items & 9.24 & 7.23 & 0.053 \\
\hline Total score & 33.47 & 25.00 & $0.015^{* *}$ \\
\hline
\end{tabular}

ALSFRS-R, ALS Functional Rating Scale-Revised. * Significant at $p<0.001$. ${ }^{* *}$ Significant at $p<0.05$.

ity. There was a strong positive correlation between the CPIB and intelligibility/STI scores $(r s=0.517, p=0.004)$ at $p<0.005$. There was also a strong positive correlation between the CPIB and bulbar subset scores ( $r \mathrm{~s}=0.792$, $p=0.000$ ), and between the STI and bulbar subset scores ( $r$ s $=0.789, p=0.000$ ), all 3 correlations being statistically significant at the $p<0.001$ level (Table 2 ).

\section{Dysarthria Severity and the ALSFRS-R}

There were significant differences between the dysarthria severity groups (no/mild vs. moderate/severe dysarthria) in both total scores on the ALSFRS-R $(p=0.046)$ at $p<0.05$ and bulbar function subset scores $(p=0.000)$ at $p<0.001$ (Table 3).

Communicative Participation in People with ALS
Table 4. Dysarthria severity, communicative participation, and intelligibility differences

\begin{tabular}{|c|c|c|c|}
\hline & $\begin{array}{l}\text { No/mild } \\
\text { dysarthria } \\
(n=17)\end{array}$ & $\begin{array}{l}\text { Moderate/severe } \\
\text { dysarthria } \\
(n=13)\end{array}$ & $p$ value \\
\hline CPIB sf (SD) & $63.64(5.63)$ & $46.47(8.11)$ & 0.000 \\
\hline $\operatorname{Min} / \max$ & $55.30 / 71.00$ & $34.00 / 64.20$ & \\
\hline Intelligibility (SD) & $97.61 \%(1.91)$ & $54.39 \%(39.98)^{\mathrm{a}}$ & 0.000 \\
\hline $\min / \max$ & $93.78 \% / 100 \%$ & $3.11 \% / 99.11 \%$ & \\
\hline
\end{tabular}

CPIB sf, Communicative Participation Item Bank short form.

${ }^{\text {a }} n=12$ because STI data were missing for 1 participant.

Dysarthria Severity, CPIB, and Speech Intelligibility

The results show that there were significant differences between the severity groups (no/mild vs. moderate/severe dysarthria) in both perceived communicative participation $(p=0.000)$ and speech intelligibility $(p=0.000)$ at $p<0.001$ (Table 4 ).

\section{Discussion}

In summary, the hypothesis that CPIB scores correlated with both bulbar deficits caused by ALS and speech intelligibility was corroborated. The correlation between speech intelligibility and the ALSFRS-R bulbar function 
subset seems intuitive, with 1 of the 3 items directly concerning speech (the item options being: normal speech processes/detectable speech disturbance/intelligible with repeating/speech combined with nonvocal communication/loss of useful speech). The CPIB scores correlated more strongly with the bulbar function subset than with the speech intelligibility scores, for which the correlation was more moderate. One rationale for this could be that the other items included in the bulbar ALSFRS-R, i.e., salivation and swallowing, capture factors other than speech intelligibility that may also impact communicative participation, e.g., excessive saliva and occasional choking.

The range of speech intelligibility in the group with moderate/severe dysarthria was very broad, as a few individuals rated to have moderate dysarthria in text reading could still elicit almost perfect intelligibility scores in nonsense sentence transcriptions. This may reflect strategies chosen by the speakers. For example, very slow speech may be more intelligible but is less natural and therefore results in a higher severity rating. As for severity levels of dysarthria, there was a significant difference between groups for bulbar items, as expected, with bulbar deficits reflected in the ratings of the listening panel. The difference between groups for the total score on ALSFRS$\mathrm{R}$ was also significant, although not as strong. There were significant differences in scores between groups for intelligibility and the CPIB.

In its present form, the CPIB is validated for people using speech as their primary mode of communication, and so the results in this study were calculated for this mode of communication. However, it was found that the 2 participants who had transitioned to communicating mainly through AAC felt very restricted in their communicative participation when they had to rely on speech, but often did not feel restricted at all when they had access to their AAC. They and other participants also mentioned a dependence on communication partners paying attention to them, and on emotional rapport. Perceived social support, i.e., the expectation that others will provide support if needed, can serve as both a facilitator of and a barrier to communication success [30]. For example, one person, who had quite mild dysarthria, felt that her communicative participation plummeted when she had to deal with the social security agency because of her administrator's negative attitude.

ALS is a disease with many different manifestations. Symptoms and progression vary greatly, but most people with ALS will experience dysarthria and lowered communicative participation. A variety of factors may have an impact on communicative participation and cannot be separated from information about communicative participation, per se, collected by means of the CPIB alone. To find out how personal or environmental factors play into the individual's experiences of interference with communicative participation, additional knowledge about and/or questions to the respondent are required [20]. When the CPIB is completed in the company of the SLP, the underlying reasoning behind the patient's answers can be discussed and help direct further services.

\section{Limitations}

Investigation of communicative participation in this population is highly relevant, but the results of this study can only be tentative, for a number of reasons (1) In a DIF analysis, the DIF was considered negligible for multiple sclerosis, $\mathrm{PD}$, and head and neck cancer, meaning that the CPIB can be used without meaningful differences between these diagnostic groups. However, because of the limited number of respondents at the time, ALS was not included in this DIF analysis [20]. (2) A DIF analysis that could show whether there is a cross-cultural response bias when comparing respondents from the USA and Sweden has not yet been completed. (3) The ALS patient base in Sweden is proportionally small. The participants in this study were drafted from the patient base in the western region of Sweden and represent all stages and types of ALS, with the exception of people with frontotemporal dementia or cognitive deficits and persons in palliative care, but the group size was still small. (4) The participants include persons with severe dysarthria, for which the CPIB has not been tested. (5) Some individuals had great problems participating physically or communicatively in a number of life situations without being placed in institutional care, thus possibly confounding the definition of "community dwellers." With these caveats in mind, this study may still show that it should be both possible and clinically valid to include the CPIB into the clinical toolbox as a means to rate and discuss communicative participation with people with ALS.

\section{Future Research}

The developers of the CPIB have pointed out the need for caution in using the CPIB with populations that differ from the one for which it has been tested, i.e., the relevance and acceptability of the CPIB items have not yet been tested for people who depend solely on AAC for communication [20]. In ALS, the progress of the disease makes the use of AAC a question of when, rather than whether, to begin using it. In the bulbar subgroup, a communication device is sometimes the first medical aid pre- 
scribed. The developers of the CPIB comment that validating the CPIB for people who rely primarily on augmentative communication "would be an important step in the development of this instrument" [17] (p. 1795). Testing to see whether the items in the CPIB can be used in their present form, maybe with an alternative instruction to allow for the use of alternative and/or augmentative communication, seems urgent for this population. If that is inappropriate, there may be a need for a CPIBAAC.

\section{Acknowledgements}

The authors would like to thank the participants with ALS as well as the listening panel who took part in this study.

\section{Statement of Ethics}

This study was approved by the Central Ethics Review Board in Stockholm, Sweden, and conducted in accordance with the Declaration of Helsinki. Written informed consent was obtained from all participants. All who were approached about participating were informed that it was voluntary and that those who chose to participate could withdraw at any time. A decision to not take part or to withdraw would have no effect on any care they received. A few individuals declined to participate, explaining that they found it hard to talk about their illness. Some of those who did participate found it was a relief to talk about it. All of those approached had ongoing contact with an ALS team and access to speech therapy and counseling. There were no personal gains, and it did impinge on participants' time and energy. To minimize fatigue and amplify comfort and control, the participants could choose to complete their interviews in their homes. The speech of 2 participants had deteriorated at the time of testing so that oral speech was no longer their primary mode of communication, but they were not excluded. Given the expected progression of speech difficulties and in recognition of the effort they had made to participate, their data were included and represent later stages of the disease.

\section{Disclosure Statement}

The authors have no conflicts of interest to declare.

\section{Funding Sources}

This study was supported by grants from FoU Region Västra Götaland.

\section{Author Contributions}

All authors participated in the design of the study as well as the analysis and interpretation of the data. The first author was responsible for participant recruitment and data collection and entry. All authors participated in discussions regarding the results and collaborated in the writing of the paper.

\section{References}

1 Andersen PM, Abrahams S, Borasio GD, de Carvalho M, Chio A, Van Damme P, et al.; EFNS Task Force on Diagnosis and Management of Amyotrophic Lateral Sclerosis. EFNS guidelines on the clinical management of amyotrophic lateral sclerosis (MALS)-revised report of an EFNS task force. Eur J Neurol. 2012 Mar; 19(3):360-75.

2 Wijesekera LC, Leigh PN. Amyotrophic lateral sclerosis. Orphanet J Rare Dis. 2009 Feb; 4(3):3.

3 Tomik B, Guiloff RJ. Dysarthria in amyotrophic lateral sclerosis: A review. Amyotroph Lateral Scler. 2010;11(1-2):4-15.

4 Beukelman D, Fager S, Nordness A. Communication support for people with ALS. Neurol Res Int. 2011;2011:714693.

5 World Health Organization. ICF: International Classification of Functioning, Disability and Health. Geneva, Switzerland: WHO; 2001.

6 Ustün TB, Chatterji S, Bickenbach J, Kostanjsek N, Schneider M. The International Classification of Functioning, Disability and Health: a new tool for understanding disability and health. Disabil Rehabil. 2003 Jun; 25(11-12):565-71.
7 Dykstra A. Communicative participation and speech intelligibility: complexities and challenges [Commentary]. Dev Med Child Neurol. 2013 May;55(5):403-4.

8 Eadie TL, Yorkston KM, Klasner ER, Dudgeon BJ, Deitz JC, Baylor CR, et al. Measuring communicative participation: a review of selfreport instruments in speech-language pathology. Am J Speech Lang Pathol. 2006 Nov; 15(4):307-20.

9 Yorkston KM, Beukelman DR, Strand EA, Bell KR. Management of Motor Speech Disorders in Children and Adults. Austin: ProEd; 1999.

10 Miller N. Measuring up to speech intelligibility. Int J Lang Commun Disord. 2013 NovDec;48(6):601-12.

11 Sussman JE, Tjaden K. Perceptual measures of speech from individuals with Parkinson's disease and multiple sclerosis: intelligibility and beyond. J Speech Lang Hear Res. 2012 Aug;55(4):1208-19.

12 Dykstra A [Internet]. What is communicative participation? Western University, Canada: 2013 July 8. Available from: http://www.uwo. $\mathrm{ca} / \mathrm{fhs} / \mathrm{cpl} /$ whatis.html
13 Baylor C, Burns M, Eadie T, Britton D, Yorkston K. A qualitative study of interference with communicative participation across communication disorders in adults. Am J Speech Lang Pathol. 2011 Nov;20(4): 269-87.

14 Hartelius L, Elmberg M, Holm R, Lövberg AS, Nikolaidis S. Living with dysarthria: evaluation of a self-report questionnaire. Folia Phoniatr Logop. 2008;60(1):11-9.

15 McAuliffe MJ, Baylor CR, Yorkston KM Variables associated with communicative participation in Parkinson's disease and its relationship to measures of health-related quality-of-life. Int J Speech Lang Pathol. 2017 Aug; 19(4):407-17.

16 Yorkston KM, Baylor C, Amtmann D. Communicative participation restrictions in multiple sclerosis: associated variables and correlation with social functioning. J Commun Disord. 2014 Nov-Dec;52:196-206.

17 Yorkston K, Baylor C, Mach H. Factors Associated With Communicative Participation in Amyotrophic Lateral Sclerosis. J Speech Lang Hear Res. 2017 Jun;60(6S Suppl):17917. 
18 Baylor C, Hula W, Donovan NJ, Doyle PJ, Kendall D, Yorkston K. An introduction to item response theory and Rasch models for speech-language pathologists. Am J Speech Lang Pathol. 2011 Aug;20(3):243-59.

19 Baylor CR, Yorkston KM, Eadie TL, Miller RM, Amtmann D. Developing the communicative participation item bank: rasch analysis results from a spasmodic dysphonia sample. J Speech Lang Hear Res. 2009 Oct;52(5):130220.

20 Baylor C, Yorkston K, Eadie T, Kim J, Chung $\mathrm{H}$, Amtmann D. The Communicative Participation Item Bank (CPIB): item bank calibration and development of a disorder-generic short form. J Speech Lang Hear Res. 2013 Aug;56(4):1190-208.

21 Baylor C. "I'll Have What He's Having..." and Other Restrictions in Communicative Participation. JAMA Otolaryngol Head Neck Surg. 2018 Aug;144(8):693-5.

22 Nordberg M, Syrjäkylä ML. Validation of translation into Swedish of Communicative Participation Item Bank by cognitive interviews with persons with MS or ataxia. Unpublished graduate paper. Stockholm: Karolinska Institutet, Department of Clinical Science, In- tervention and Technology (CLINTEC) Division of Speech and Language Pathology. Available from: https://clintec.ki.se/Exam_ logopedi/pdf/355.pdf

23 Mellenthin L, Roos E. Validation of the Swedish translation of Communicative Participation Item Bank on individuals with Parkinson's disease or atypical Parkinsonism. Unpublished graduate paper. Gothenburg: University of Gothenburg, Speech and Language Pathology Unit; 2013., available from https://core.ac.uk/display/18175394

24 Baylor C, McAuliffe MJ, Hughes LE, Yorkston $\mathrm{K}$, Anderson T, Kim J, et al. A differential item functioning (DIF) analysis of the Communicative Participation Item Bank (CPIB): comparing individuals with Parkinson's disease from the United States and New Zealand. J Speech Lang Hear Res. 2014 Feb;57(1):90-5.

25 Cedarbaum JM, Stambler N, Malta E, Fuller C, Hilt D, Thurmond B, et al.; BDNF ALS Study Group (Phase III). The ALSFRS-R: a revised ALS functional rating scale that incorporates assessments of respiratory function. J Neurol Sci. 1999 Oct;169(1-2):13-21.

26 The Amyotrophic Lateral Sclerosis Functional Rating Scale. Assessment of activities of dai- ly living in patients with amyotrophic lateral sclerosis. The ALS CNTF treatment study (ACTS) phase I-II Study Group. Arch Neurol. 1996 Feb;53(2):141-7.

27 Bacci ED, Staniewska D, Coyne KS, Boyer S, White LA, Zach N, et al.; Pooled Resource Open-Access ALS Clinical Trials Consortium. Item response theory analysis of the Amyotrophic Lateral Sclerosis Functional Rating Scale-Revised in the Pooled Resource Open-Access ALS Clinical Trials Database. Amyotroph Lateral Scler Frontotemporal Degener. 2016;17(3-4):157-67.

28 Ozanne AG, Persson LI. Correlations in health status between estimates of families of people with amyotrophic lateral sclerosis and estimates of staff. Palliat Support Care. 2013 Jun;11(3):183-9.

29 Hartelius L, Svensson P. Dysartritest. Stockholm: Psykologiförlaget AB; 1990.

30 Eadie T, Kapsner-Smith M, Bolt S, Sauder C, Yorkston K, Baylor C. Relationship between perceived social support and patient-reported communication outcomes across communication disorders: a systematic review. Int J Lang Commun Disord. 2018 Nov;53(6): 1059-77. 